\title{
Study on Vehicle Instability Control Based on Hydraulic coordinator of Electronic Stability Program and Anti-lock Brake System
}

\author{
Chen Song ${ }^{1, ~ a, ~ L i ~ S h e n g y o n g ~}{ }^{1, b}$, Zhang Zhihua ${ }^{1, c}$ \\ ${ }^{1}$ Department of Transportation Engineering, Nantong Shipping College, Nantong 226010, China \\ achensong@ntsc.edu.cn, 'isy@ntsc.edu.cn, 'czhangzh@ntsc.edu.cn
}

\begin{abstract}
Keywords: Hydraulic coordinator, Electronic Stability Program, Anti-roll Brake System, Sliding mode, Coordinate, Instability
\end{abstract}

\begin{abstract}
Since a vehicle is prone to instability under extreme steering working conditions, an Electronic Stability Program (ESP for short) is adopted. The dynamic characteristics of the vehicle are nonlinear under the extreme conditions. To control the nonlinearity, a sliding mode controller is established based on the sliding mode theory with ESP. Because the ESP control of vehicle instability is carried out by the method of differential braking, the reducing of the lateral force provided by the tires will be caused due to the wheels locking. To prevent the lock of the wheels, an anti-roll brake system (ABS for short) control system is integrated with ESP. And considering the control confliction of the ESP and the ABS, a hydraulic coordinator is designed to mitigate their confliction. Correspondingly, the control rules of the ESP and the hydraulic coordinator for vehicle instability prevention are set. Lastly, simulation experiments with the proposed control system are conducted under the step working condition. The simulation results show that the controller of ESP and ABS with the confliction hydraulic coordinator can prevent the vehicle rollover, and enhance the driving stability of a vehicle effectively.
\end{abstract}

\section{Introduction}

The loss of yaw stability will lead to the loss of steering capability or will cause a fierce turning of a vehicle. At present, the relatively mature ESP system on the market is mainly carried out by the method of differential braking to control instability. Differential braking control can produce an additional yaw moment by putting different braking force on different wheels, and then realize the prevention of the vehicle instability. In former studies, many modern control methods, such as model predictive control, optimal control, and fuzzy PID control, have been applied to improve the control effect of the ESP system. However, as a vehicle system including multiple control units, there is little research on the interaction between other systems when using ESP to control instability. Although some literatures have also pointed out that the impact between ESP and ABS needs considering, most of them do not propose an explicit method for specific research. In this paper, considering the control effect of the vehicle stability during wheel locking caused by braking, the integration of ESP and ABS is proposed. In order to resolve the conflict between the ESP and ABS systems, a hydraulic coordinator is designed to meet the control requirements of both without changing their original controller.

\section{Vehicle Dynamic Model}

Taking the vehicle with ESP and ABS systems as the research object, the eight-degree-of-freedom dynamic model is established for a vehicle. As shown in Fig. 1, this model contains the yaw motion, the roll motion, the longitudinal motion, the lateral motion and four wheels rotate motions of the vehicle body. 

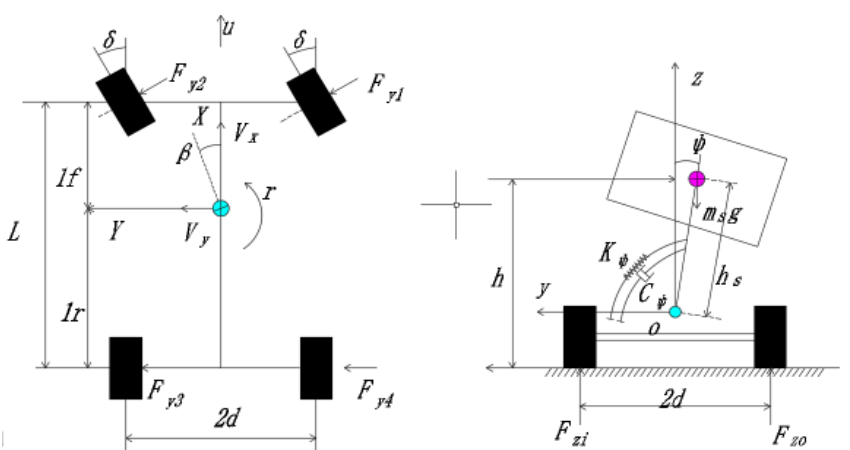

Fig. 1 DOF the vehicle dynamic model.

The equation of longitudinal motion:

$m\left(\dot{v}_{x}-v_{y} \gamma\right)=\left(F_{x 1}+F_{x 2}\right) \cos \delta-\left(F_{y 1}+F_{y 2}\right) \sin \delta+F_{x 3}+F_{x 4}$

The equation of lateral motion:

$m\left(\dot{v}_{y}+v_{x} \gamma\right)-\mathrm{m}_{\mathrm{s}} \mathrm{h} \ddot{\varphi}=\left(F_{y 1}+F_{y 2}\right) \cos \delta+F_{y 3}+F_{y 4}$

The equation of yaw motion:

$I_{Z} \dot{\gamma}+I_{X Z} \ddot{\varphi}=l_{f}\left(F_{y 1}+F_{y 2}\right) \cos \delta-l_{r}\left(F_{y 3}+F_{y 4}\right)+M$

The equation of roll motion:

$$
I_{x} \ddot{\varphi}-m_{s} h_{s}\left(\dot{v}_{y}+v_{x} \gamma\right)+I_{x z s} \dot{\gamma}=m_{s} g h_{s} \varphi-k_{\varphi} \varphi-c_{\varphi} \dot{\varphi}
$$

For the influence of vehicle vertical load transfer and nonlinear characteristics of tires when vehicle steering, the lateral force $F_{y i}(\mathrm{i}=1,2,3,4)$ and longitudinal force $F_{x i}(\mathrm{i}=1,2,3,4)$ can be inferred by Magic Tire model. The symbols of the above expressions and Fig. 1 are found in Table 1.

Table 1. The symbols in Fig. 1 and the above expressions.

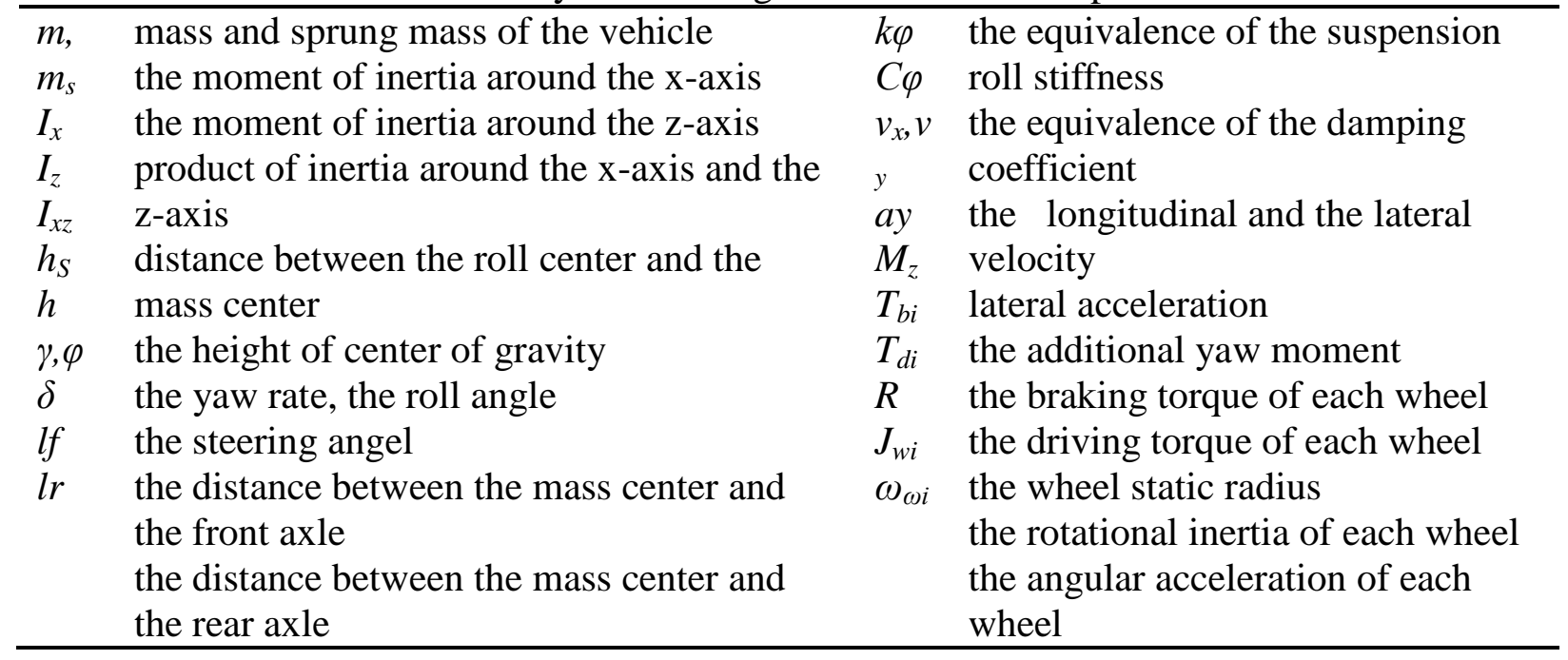

\section{The Design of Sliding Mode Controller}

The two-degree-freedom dynamic model in this paper is shown as follow:

$$
\left(k_{1}+k_{2}\right) \beta+\frac{1}{u}\left(l_{f} k_{1}-l_{r} k_{2}\right) w_{r}-k_{1} \delta=m u\left(\dot{\beta}+w_{r}\right)
$$




$$
\left(l_{f} k_{1}-l_{r} k_{2}\right) \beta+\frac{1}{u}\left(l_{f}^{2} k_{1}+l_{r}^{2} k_{2}\right) w_{r}-l_{f} k_{1} \delta=I_{Z} \dot{w}_{r}
$$

To increase the stability margin of the vehicle, it is often desired that the response of vehicle side-slip angle $\beta$ tends to zero [1]. Formula (6) shows that when the vehicle side-slip angle tends to zero, the yaw rate is the first-order lag response of the front wheel angle. So, Formula (7) is derived by making a Laplace transform of Formula (6).

$$
\frac{\gamma}{\delta}(s)=G_{r} \frac{1}{1+T_{e} s}
$$

In the above expression, $G_{r}=\frac{1}{1+A u^{2}} \cdot \frac{u}{l}$. In the expression of $G r, A=\frac{m}{l^{2}} \cdot \frac{l_{r} c_{r}-l_{f} c_{f}}{c_{f} \cdot c_{r}}$, and $T_{e}=\frac{1}{\sqrt{p}}$, where $p=\frac{l^{2} c_{f} c_{r}}{m I u^{2}}\left(1+A u^{2}\right)$. The formula (7) is transformed into (8).

$$
\delta G_{r}=\gamma+T_{e} \gamma S
$$

Formula (11) can be derived from the Laplace inverse transform of Formula (8).

$$
-\delta G_{r}+\gamma+T_{e} \dot{\gamma}=0
$$

When the vehicle is unstable, due to the influence of vehicle load transfer on tire cornering, the dynamic characteristic of the vehicle is complex nonlinear. As a kind of nonlinear control, sliding mode variable structure control switches the control size and symbols in the ideal way in different control area based on the current state, the deviation, and the derivative value of the system, making the system do small and high-frequency back and forth movement along the defined state trajectory under certain conditions, until the motion of the system state becomes a slip along the switching line. Because the given phase trajectory has nothing to do with the change of control object parameters and external disturbance, when the system moves on the sliding surface, the sliding mode control has a more superior robustness to that of other control systems. From the above analysis, it can be concluded that the sliding mode control is fit for the control of instability vehicle [2]. The sliding mode surface:

$$
S=T_{e} \dot{\gamma}+\gamma-G_{r} \delta=0
$$

For this sliding surface to have stable dynamics, condition (11) must be satisfied.

$$
\dot{S}+k S=0(k>0)
$$

In the formula, $K$ is the sliding mode control parameter. Formula (12) can be obtained by substituting Formula (10) into Formula (11).

$$
T_{e} \ddot{\gamma}+\left(1+k T_{e}\right) \dot{\gamma}+k \gamma-G_{r} \dot{\delta}-k G_{r} \delta=0
$$

From the complex function $\ddot{\gamma}(s)=s \dot{\gamma}(s)$, it can be achieved as follow:

$$
\dot{\gamma}=\frac{1}{T_{e} s+1+k T_{e}}\left(G_{r} \dot{\delta}+k G_{r} \delta-k \gamma\right)
$$

Rearranging (3), the control yaw moment $\Delta M$ is expressed as in (14).

$$
M=I_{Z} \dot{\gamma}+I_{X Z} \ddot{\varphi}-\left[l_{f}\left(F_{y 1}+F_{y 2}\right) \cos \delta-l_{r}\left(F_{y 3}+F_{y 4}\right)\right]
$$

Substituting Formula (15) into Formula (16), the control yaw moment $\Delta M$ turns into Formula (15). 


$$
M=I_{Z} \frac{1}{T_{e} s+1+k T_{e}}\left(G_{r} \dot{\delta}+k G_{r} \delta-k \gamma\right)+I_{X Z} \ddot{\varphi}-\left[l_{f}\left(F_{y 1}+F_{y 2}\right) \cos \delta-l_{r}\left(F_{y 3}+F_{y 4}\right)\right]
$$

ESP and ABS Coordinator. When the braking is put on different wheels of an unstable vehicle, the effects of the vehicle stability control are different. If a vehicle over-steers, to control the front-outside wheel achieves the best effect; if a vehicle under-steers, to control the rear-inner wheel achieves the best effect [3]. As for the instability control by differential braking, the biggest drawback is to produce the undesired deceleration of the driver. Therefore, to meet the driver's expectation, it is better to use the single-wheel braking. Based on that criterion and combined with the control efficiency of the wheel braking, this paper, in terms of the vehicle steering characteristic, adopts to brake a single wheel firstly, whose control efficiency is the best, to control the vehicle instability. And in order to prevent the wheel locking in the braking, this paper integrates ABS system with ESP. when the slip ratio of the wheel is lower than the lower limit of the ABS control, the ABS will pressurize the brake wheel cylinder; when the slip ratio of the wheel is higher than the upper limit of the ABS control, the ABS will depressurize the brake wheel cylinder; when the slip ratio is between the upper and lower limit, the original output mode will be unchanged, and the ABS will keep a constant brake wheel cylinder pressure. When the slip ratio of the wheel exceeds the value of the ABS control threshold in the vehicle instability control, the ESP and ABS hydraulic coordinator is needed to guarantee the effect of yaw stability control without changing the original ABS function, and a differential braking hydraulic coordinator is established to redistribute the braking force. The control idea of the hydraulic coordinator is: when the ABS works and keeps the slip ratio of tires near the target value, the variation of the yaw moment caused by the increase or the decrease of the brake pressure is $\Delta M i$. Because the yaw moment produced by the controlled wheel cannot meet the instability control requirement of the ESP system of vehicle due to the existence of the yawing moment $\Delta M i$, the coordinate controller is needed to redistribute the braking force of the each wheel to offset the varying yaw moment $\Delta M i$, satisfying the yaw moment required to control vehicle instability. Then, the coordinate controller can satisfy the control requirements of both the ABS and the ESP systems at the same time. Combined with the experience of the literature [4] and [5], the control rules of the ESP and the hydraulic coordinator for vehicle instability prevention are shown in Table 2.

Table 2. Control rules of the ESP and the coordinator.

\begin{tabular}{|c|c|c|c|c|c|}
\hline \begin{tabular}{|c|}
$\begin{array}{c}\text { Steering } \\
\text { angle }\end{array}$ \\
\end{tabular} & $\begin{array}{c}\text { Yaw rate } \\
\text { difference }\end{array}$ & \begin{tabular}{|c|}
$\begin{array}{c}\text { Vehicle } \\
\text { state }\end{array}$ \\
\end{tabular} & $\begin{array}{c}\text { ESP } \\
\text { control } \\
\end{array}$ & ABS control & Coordinator control \\
\hline \multirow{2}{*}{$\delta>0$} & \multirow{2}{*}{$\Delta \gamma \geq c_{1} \gamma_{N o}$} & \multirow{2}{*}{$\begin{array}{c}\text { Over- } \\
\text { steering }\end{array}$} & \multirow{2}{*}{$\begin{array}{c}\text { Front-right } \\
\text { wheel }\end{array}$} & $\mathrm{s}>\mathrm{s}_{\text {ceiling, }}$ decrease pressure & Rear-right wheel increase pressure in cylinder \\
\hline & & & & $\mathrm{s}<\mathrm{s}_{\text {floor }}$, increase pressure & Increase pressure in front-left wheel cylinder \\
\hline \multirow{2}{*}{$\delta<0$} & \multirow{2}{*}{$\Delta \gamma \leq-c_{1} \gamma_{N o}$} & \multirow{2}{*}{\begin{tabular}{|c|} 
Over- \\
steering \\
\end{tabular}} & \multirow{2}{*}{$\begin{array}{c}\text { Front-left } \\
\text { wheel }\end{array}$} & $\mathrm{s}>\mathrm{s}_{\text {ceiling, }}$ decrease pressure & Increase pressure in rear-left wheel cylinder \\
\hline & & & & $\mathrm{s}<\mathrm{s}_{\text {floor, }}$, increase pressure & Increase pressure in front-right wheel cylinder \\
\hline \multirow{2}{*}{$\delta<0$} & \multirow{2}{*}{$\Delta \gamma \geq c_{1} \gamma_{N o}$} & \multirow{2}{*}{\begin{tabular}{|c|} 
Under- \\
steering \\
\end{tabular}} & \multirow{2}{*}{$\begin{array}{c}\text { Rear-right } \\
\text { wheel }\end{array}$} & $\mathrm{s}>\mathrm{S}_{\text {ceiling, }}$ decrease pressure & Increase pressure in front-right wheel cylinder \\
\hline & & & & $\mathrm{s}<\mathrm{s}_{\text {floor, }}$, increase pressure & Increase pressure in front-left wheel cylinder \\
\hline \multirow{2}{*}{$\delta>0$} & \multirow{2}{*}{$\Delta \gamma \leq-c_{1} \gamma_{N o}$} & \multirow{2}{*}{\begin{tabular}{|c|} 
Under- \\
steering \\
\end{tabular}} & \multirow{2}{*}{$\begin{array}{c}\text { Rear-left } \\
\text { wheel }\end{array}$} & $\mathrm{s}>\mathrm{s}_{\text {ceiling, }}$ decrease pressure & Increase pressure in front-left wheel cylinder \\
\hline & & & & $\mathrm{s}<\mathrm{s}_{\text {floor, }}$ increase pressure & Increase pressure in front-right wheel cylinder \\
\hline random $\delta$ & $-c_{1} \gamma_{N}<\Delta \gamma<c \gamma_{N b}$ & stability & none & No control & No control \\
\hline
\end{tabular}

\section{Simulations and Results Analysis}

To verify the control effect on vehicle instability based on the coordinating control of ESP and ABS under the limiting steering condition, this paper adopts the step simulation test. The simulation step conditions are like these: the attachment coefficient of the road surface is 0.8 ; and the vehicle makes a uniform straight line driving with a velocity of $80 \mathrm{~km} / \mathrm{h}$. For comparison, a passive system without any control (be shown as Open Loop in the figure), an ESP system without integration of ABS (be shown as ESP without ABS in the figure), and an ESP system integrated with ABS (be shown as ESP with $A B S$ in the figure), have been carried out respectively in simulation. From Fig. (2) and (3), it can be seen that without any control the yaw rate and side-slip angle of the vehicle are 
larger and the oscillation time is longer, and the vehicle cannot return to steady state for a long time. From Fig. (4), it is found that there is a steady focus in the uncontrolled phase plane curve, but the vehicle side-slip angle changes greatly, and the vehicle will lose stability, drifting and turning fiercely. The overshoots of the yaw rate and the side-slip angle with the coordinating control of ESP and ABS are smaller than the uncontrolled ones; the responses are quicker; and the values can quickly get back to the stable region, thus effectively controlling the instability of the vehicle.

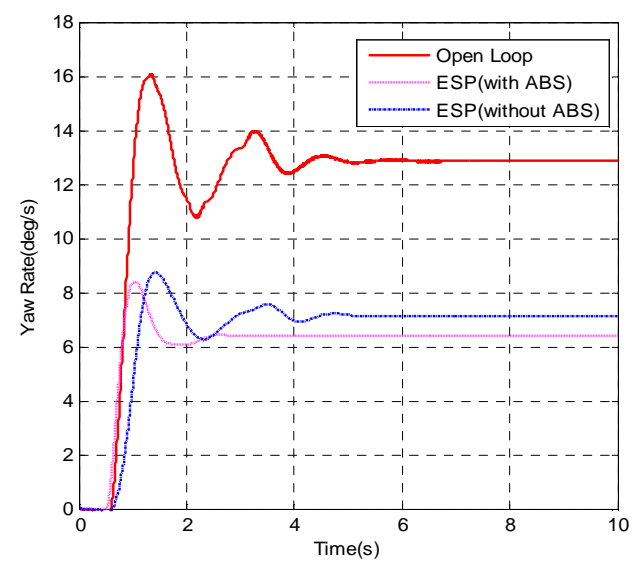

Fig. 2 Yaw rate response curve.

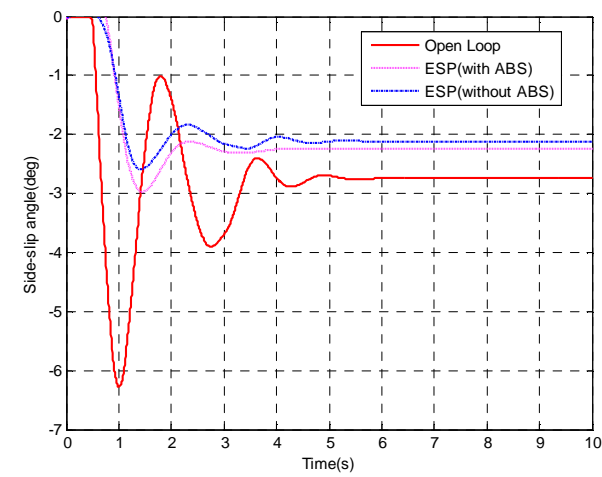

Fig. 3 Side-slip angle response curve.

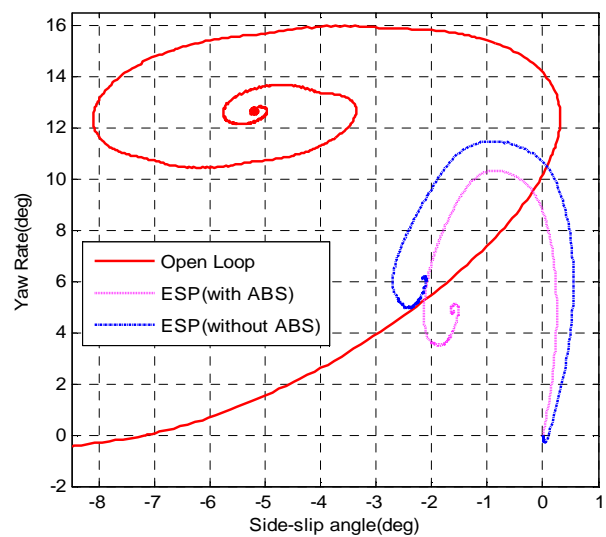

Fig. 4 Phase diagram of side-slip angle and yaw rate.

The ESP system achieves the stability control by braking the wheel with the highest efficiency of vehicle stability control. From Fig. 5 (a) and 5 (b), and Fig. 6 (a) and 6 (b), the ESP system performs brake control on the front-right wheel (FR in the figure) of the vehicle due to the excessive steering of the vehicle, and the other wheels are not involved in braking, so only the braking force and the slip rate of the outer front wheel are not zero, and those of the rest wheels braking force and slip rate are zero. While the integrated system of ESP and ABS, adjusting the 
brake oil pressure to control the wheel with the highest stability efficiency of the vehicle and also controlling the side-slip rate of the wheel through ABS, keeps the slip ratio of the wheel between $8 \%$ and $35 \%$. In order to provide the yaw moment required to maintain the vehicle stability when ABS is controlling the value of side-slip ratio, the coordinated controller performs the braking control on the rear-right wheel (RR in the figure). So, the values of brake oil pressure of the front-right wheel and the rear-right wheel of the vehicle with integrated control of ESP and ABS are not zero and are less than that simply controlled with ESP. Compared to the range of wheel slip ratio of the vehicle using the ESP control alone, that under the integrated control of ESP and ABS is in the more favorable range to the control the vehicle stability. Therefore, the integrated system of ESP and ABS is superior to the ESP control system without ABS in the yaw control.

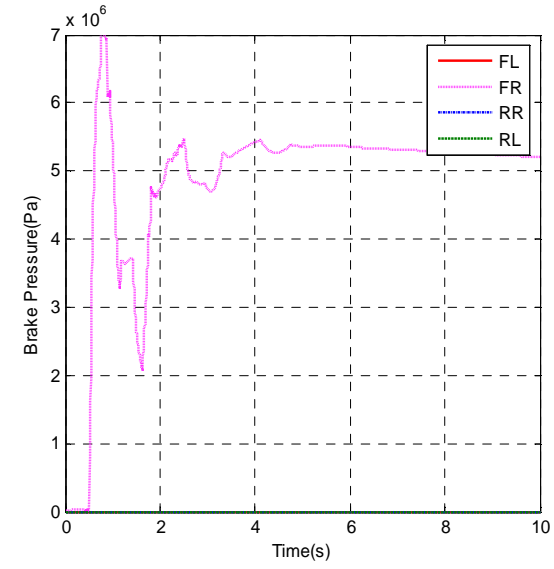

Fig. 5(a) Braking pressures of each wheel (without ABS).

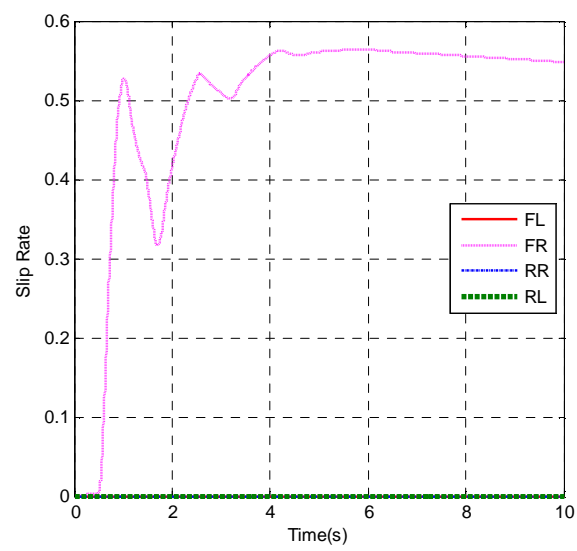

Fig. 6(a) Slip ratios of each wheel (without ABS).

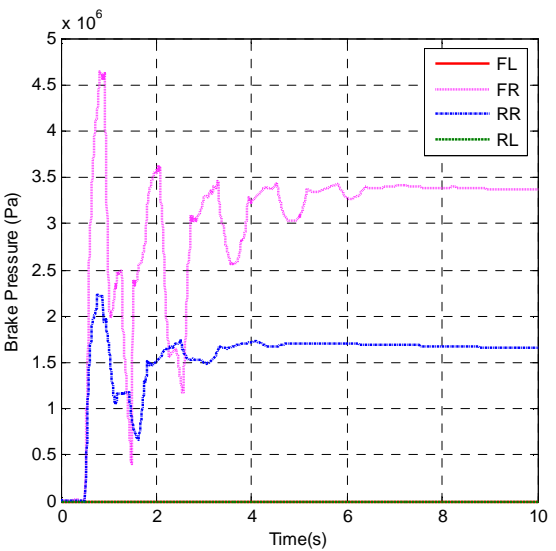

Fig. 5(b) Braking pressures of each wheel (with ABS).

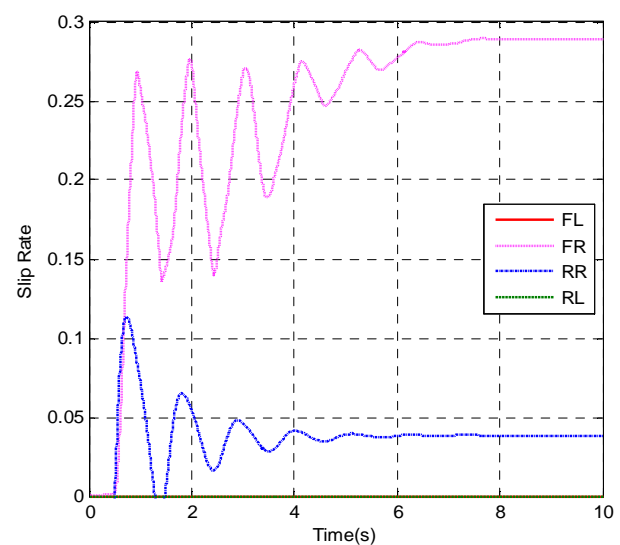

Fig. 6(b) Slip ratios of each wheel (with ABS).

\section{Conclusion}

(1) Since a vehicle is prone to instability under extreme steering working conditions, a sliding mode controller, employing ESP system, is designed in this paper.

(2) To ensure the control effect of vehicle stability, an ABS control system is integrated with ESP system. And to avoid the control confliction of the ESP and the ABS, a coordinator is designed to meet their own control requirements.

(3) The simulation results show that the controller of ESP and ABS with the confliction coordinator can prevent the vehicle rollover, and can enhance the driving stability of a vehicle effectively. 


\section{Acknowledgement}

In this paper, the research was supported Research innovation projects of Jiangsu College graduate (grant no. CXLX12_0629).

\section{References}

[1] Y. H. Chen, J. K. Hedrick, K. H. Cuo. A novel direct yaw moment controller for in-wheel motor electric vehicles. Vehic. Syst. Dyn. 51(6) (2013) 925-942.

[2] K. Byunghak. Robust vehicle stability controller by multiple sliding mode control. AVEC'00, 2000 .

[3] O. Barbarisi, G. Palmieri, S. Scala, et al. LTV-MPC for yaw rate control and side slip control with dynamically constrained differential Braking. European J. Contr. 15(3) (2009) 468-479.

[4] S. Antonov, A. Fehn, A. Kugi, Unscented Kalman Filter for Vehicle State Estimation, Vehic. Syst. Dyn. 49(9) (2009) 1497-1520.

[5] R. Rajamani. Vehicle dynamics and control. Springer-Verlag New York Inc. (2005), pp. 201-235. 